\title{
Pressure-driven miscible two-fluid channel flow with density gradients
}

\author{
K. C. Sahu, H. Ding, P. Valluri and O. K. Matar* \\ Department of Chemical Engineering, \\ Imperial College London, SW7 2AZ, UK
}

(Dated: November 5, 2008)

\begin{abstract}
We study the effect of buoyancy on pressure-driven flow of two miscible fluids in inclined channels via direct numerical simulations. The flow dynamics are governed by the continuity and NavierStokes equations, without the Boussinesq approximation, coupled to a convective-diffusion equation for the concentration of the more viscous fluid through a concentration-dependent viscosity and density. The effect of varying the density ratio, Froude number, and channel inclination on the flow dynamics is examined, for moderate Reynolds numbers. We present results showing the spatio-temporal evolution of the flow together with an integral measure of mixing.
\end{abstract}

*Email: o.matar@imperial.ac.uk 


\section{INTRODUCTION}

The stability of two-fluid flows has been the subject of numerous investigations due to their relevance to practical applications; these include the transportation of crude oil in pipelines [1], mixing of liquids using centreline injectors, upstream of static mixers [2], and the removal of highly viscous or elasto-viscoplastic material adhering to pipes by using fastflowing water streams [3]. Although a large number of studies have examined the stability of immiscible systems [1,4], which have included linear stability analysis for channels [512], horizontal [13-17] and vertical pipes [18, 19], with density and viscosity contrasts, experiments [20, 21] and numerical simulations in straight [22-24] and corrugated pipes [2528], miscible systems have received far less attention. The objective of the present paper is therefore to examine the characteristics of pressure-driven two-fluid channel flow with density and viscosity contrasts using numerical simulations.

The linear stability of three-layer Poiseuille channel flows were analysed by Ranganathan and Govindarajan [29] and Govindarajan [30] who demonstrated the presence of instability at low Reynolds number and high Schmidt number. Diffusion has also been shown to drive instability for continuous but rapidly varying viscosity stratification [31]. In miscible core-annular flows, experimental studies have focused on the the thickness of the more viscous layer left on the pipe walls and the tip speed of the propagating 'finger' of the displacing, less viscous fluid [32-37]. The development of axisymmetric and "corkscrew" patterns in miscible flows have also been investigated [2, 38-41], and, axisymmetric "pearl" and "mushroom" patterns were observed in neutrally-buoyant, core-annular horizontal pipe flows at high Schmidt number and Reynolds numbers in the range $2<R e<60$ [42]. A recent linear stability of neutrally-buoyant, core-annular flows [43] showed that, beyond a critical viscosity ratio, the flow is unstable even in situations wherein the less viscous fluid is at the wall; this is in contrast to the case of immiscible lubricated pipelining [1] and miscible channel flows [44].

Related studies of miscible flows have focused on the effect of buoyancy in inducing mixing in the context of chemical, oil-and-gas and environmental settings [45]. A typical system studied involves two, initially stratified, miscible fluids of different densities in vertical and tilted tubes, with the more dense fluid overlying the less dense one [46-50]. The velocity of the leading edge of the inter-penetration zone, and the axial and temporal evolution of 
the mean relative concentration are measured as a function of tilt angle for a given set of fluid physical properties. The results of these studies have demonstrated that for sufficiently low tilt angles, the dynamics of the mean relative concentration can be described by a diffusion equation with a reasonably well-defined diffusion coefficient. The dependence of this coefficient and of the leading edge velocity on system parameters has elucidated the interplay between the dominant physical processes involved in these flows: axial interpenetration of the fluids driven by the gravity component parallel to the axis of the tube [51]; fluid segregation driven by the gravity component transverse to the tube axis [52]; fluid mixing driven by Kelvin-Helmholtz-type instabilities [53]. With increasing tilt angle the dynamics become less diffusive, the flow being dominated by buoyancy and inertia at relatively early times, and by viscous forces at late times $[50,54]$.

The flow dynamics has also been investigated [55] using direct numerical simulations of the flow in two- and three-dimensional channels as well as tubes. The results of this work demonstrate the effect of geometry differences on the vorticity field at late times. In particular, it is shown that the vortices which develop during the flow are more coherent and persistent in two than in three dimensions. Consequently, they give rise to more intense mixing and long-lasting flow structures in two-dimensional than three-dimensional channels and cylinderical tubes.

The linear stability of three-layer neutrally-buoyant miscible channel flows at relatively large Reynolds number was more recently studied by Sahu et al. [56]. Their results identified the regions in parameter space (Reynolds and Schmidt numbers, and viscosity ratio) where the flow is either convectively or absolutely unstable. The numerical simulations of the pressure-driven displacement of a highly viscous fluid by a less viscous one carried out by Sahu et al. [56] demonstrated the development of Kelvin-Helmholtz instabilities; these became more pronounced with increasing viscosity ratio, Reynolds and Schmidt numbers, leading to more intense mixing and rapid removal of the more viscous fluid.

In spite of the large number of studies carried out on miscible two-fluid flows, very few studies have examined the effect of density contrasts on pressure-driven displacements in tilted tubes at moderate to large Reynolds numbers; this is the subject of the present work. We build on the work of Sahu et al. [56] and solve the continuity and Navier-Stokes equations in a two-dimensional channel coupled to a convective-diffusion equation for the concentration of the more viscous fluid through a concentration-dependent viscosity and density. Details 
of the problem formulation and the numerical technique used to carry out the computations are given in section II. We investigate the effect of the density ratio, Froude number, and channel inclination on the flow characteristics and the rate of displacement of the more viscous phase. A discussion of our results is provided in section III, and concluding remarks in section IV.

\section{FORMULATION}

\section{A. Governing equations}

We consider the flow of two miscible, Newtonian and incompressible fluids of varying viscosity and density in a planar channel, inclined at an angle $\theta$ to the horizontal. We focus on the displacement of the more viscous fluid, which occupies the channel initially, by the less viscous one. We use a rectangular coordinate system $(x, y)$ to model the flow dynamics where $x$ and $y$ denote the horizontal and vertical coordinates, respectively, as shown in Fig. 1. The channel inlet and outlet are located at $x=0$ and $x=L$, and its rigid and impermeable walls at $y=0$ and $y=H$, respectively.

In order to determine the flow characteristics, we solve the continuity and Navier-Stokes equations, and a convective-diffusion equation for the concentration of the more viscous fluid. Solutions of these equations are subject to no-slip, no-penetration and no-flux conditions at $y=(0, H)$. We impose a fully-developed velocity profile with a constant flow rate at the inlet $(x=0)$, and Neumann boundary conditions at the outlet $(x=L)$. Initially the channel is occupied by a stationary, viscous fluid with the concentration, $c$, set to unity. This is then pushed by a fluid of lower viscosity from the channel inlet.

The following scaling is employed in order to render these equations dimensionless:

$$
\begin{gathered}
\mathbf{u}=\left(u_{d}, v_{d}\right)=V \mathbf{u}=V(u, v), \quad\left(x_{d}, y_{d}\right)=H(x, y), \quad t_{d}=\frac{H}{V} t, \\
p_{d}=\rho V^{2} p, \quad \rho_{d}=\rho_{2} \rho, \quad \mu_{d}=\mu_{2} \mu,
\end{gathered}
$$

where the subscript, $d$, designates dimensional quantities. In Eq. (1), u $=(u, v)$ represents the two-dimensional velocity field where $u$ and $v$ denote velocity components parallel and perpendicular to the channel; $V \equiv Q / H$ is a characteristic velocity, where $Q$ denotes the total flow rate, and $p$ and $t$ denote pressure and time, respectively. The density $\rho$ and viscosity $\mu$ have been scaled on that of the less viscous fluid, $\mu_{2}$ and $\rho_{2}$ respectively. 
The dimensionless governing equations are expressed by

$$
\begin{gathered}
\frac{\partial u}{\partial x}+\frac{\partial v}{\partial y}=0 \\
\rho\left[\frac{\partial u}{\partial t}+u \frac{\partial u}{\partial x}+v \frac{\partial u}{\partial y}\right]=-\frac{\partial p}{\partial x}+\frac{1}{R e}\left[2 \frac{\partial}{\partial x}\left(\mu \frac{\partial u}{\partial x}\right)+\frac{\partial}{\partial y}\left(\mu\left(\frac{\partial u}{\partial y}+\frac{\partial v}{\partial x}\right)\right)\right]-\frac{1}{F r^{2}} \rho \sin \theta \\
\rho\left[\frac{\partial v}{\partial t}+u \frac{\partial v}{\partial x}+v \frac{\partial v}{\partial y}\right]=-\frac{\partial p}{\partial y}+\frac{1}{R e}\left[\frac{\partial}{\partial x}\left(\mu\left(\frac{\partial u}{\partial y}+\frac{\partial v}{\partial x}\right)\right)+2 \frac{\partial}{\partial y}\left(\mu \frac{\partial v}{\partial y}\right)\right]-\frac{1}{F r^{2}} \rho \cos \theta \\
\frac{\partial c}{\partial t}+u \frac{\partial c}{\partial x}+v \frac{\partial c}{\partial y}=\frac{1}{R e S c}\left(\frac{\partial^{2} c}{\partial x^{2}}+\frac{\partial^{2} c}{\partial y^{2}}\right)
\end{gathered}
$$

where $c$ is the concentration, which represents the fraction of the channel occupied by the more viscous fluid; $R e \equiv \rho_{2} V H / \mu_{2}, F r \equiv V / \sqrt{g} H$, and $S c \equiv \mu_{2} / \rho_{2} \mathcal{D}$ denote Reynolds, Froude number and Schmidt numbers, respectively, wherein $\mathcal{D}$ denotes a constant diffusion coefficient. We assume that the dimensionless viscosity and density have the following dependence on $c$

$$
\mu=e^{c \ln (m)}, \quad \text { and } \quad \rho=c r+(1-c),
$$

where $m \equiv \mu_{1} / \mu_{2}$ and $r \equiv \rho_{1} / \rho_{2}$ are the viscosity and density ratios respectively. In the present work, we investigate the effect of $r, F r$ and $\theta$ on the dynamics and set $m=2$, $R e=200$ and $S c=100$ for the remainder of this paper; the effect of these parameters on the flow characteristics was investigated by Sahu et al. [56].

\section{B. Numerical procedure}

Here, we describe the methodology used to obtain numerical solutions of Eqs. (2)-(5). We use a staggered grid in order to discretise these equations using the finite-volume method. The scalar variables (the pressure and concentration) and the velocity components are defined at the center of each cell and at the cell faces, respectively. The solution methodology employs the following procedure: the concentration field is first updated by solving Eq. (5) with the velocity field at time steps $n$ and $n-1$; this is then updated to time-step $n+1$ by solving Eqs. (3) and (4) together with the continuity equation, Eq. (2). We use a weighted essentially non-oscillatory (WENO) scheme to discretise the advective term, the second term on the left-hand-side of Eq. (5), while a central difference scheme is used to discretize the diffusive term on the right-hand-side of Eq. (5). In order to achieve second-order accuracy 
in the temporal discretization, the Adams-Bashforth and Crank-Nicolson methods are used for the advective and second-order dissipation terms, respectively.

The numerical procedure described above has previously been employed by Ding et al. [57] to solve Eqs. (2), (3), (4) along with a Cahn-Hilliard equation for the interfacial position within the framework of the "diffuse interface" method. This procedure has been used to simulate accurately and efficiently Rayleigh-Taylor instabilities in incompressible two-phase flows with density contrasts [57], droplet spreading [58, 59], droplet deformation due to shear flow [60] in the presence of moving contact lines. Sahu et al. [56] also used this procedure to simulate the pressure-driven neutrally-buoyant, miscible channel flows with high viscosity contrasts. The results of this paper are discussed next.

\section{RESULTS AND DISCUSSION}

We begin the presentation of our results by plotting in Fig. 2a the temporal evolution of a dimensionless measure of the mass of the displaced fluid '1', $M_{0.95} / M_{0}$, for $R e=200$, $S c=100, F r=0.316, m=2, r=1.5$, and $\theta=30^{\circ}$. Here, $M_{0.95}$ and $M_{0}$ denote the mass of fluid with $c \geq 0.95$ and that of fluid ' 1 ' initially occupying the channel, respectively. The parameter values chosen are characteristic of a situation where a fluid is displaced by another, of lower density and viscosity; in this case, one would expect the flow to be destabilised due to viscous contrasts and via a Rayleigh-Taylor (RT) mechanism.

Inspection of Fig. 2a reveals that $M_{0.95} / M_{0}$ undergoes an almost linear decrease at the earlier stages of the flow due to its displacement by fluid ' 2 '. The slope of the curve during this linear stage is considerably steeper than that of the line represented by $1-t H / L$; this corresponds to the plug flow displacement of fluid ' 1 ' by fluid '2' when the sharp interface separating the fluids has not yet reached the channel exit. At approximately $t=25$ for this set of parameters when the 'front' of the displacing fluid '2 reaches the end of simulation domain, as shown in Fig. 3, a transition to another linear regime occurs; the slope of the $M_{0.95} / M_{0}$ versus time plot in this regime is much smaller than the previous one. As shown in Fig. 2b, the position of the leading 'front' separating the two fluids, $x_{\text {tip }}$, however, exhibits a linear dependence on time. It is also evident upon inspection of Fig. 2 that the results converge upon mesh-refinement.

The origin of the regime transitions in Fig. 2a may be explained by examining the flow 
dynamics. As shown in Fig. 3, which depicts the spatio-temporal evolution of the concentration contours for the same parameters as in Fig. 2, the pressure-driven displacement of fluid ' 1 ' by fluid ' 2 ' is accompanied by the development of instabilities; these manifest themselves via the formation of vortical structures, which give rise to intense mixing of the two fluids. The pressure-driven flow induces motion of the less dense, fluid ' 2 ' into the more dense, fluid ' 1 ', and is opposed by the gravitationally-driven flow, proportional to $g \sin \theta$, which accelerates fluid ' 1 ' into fluid '2'. The latter is primarily responsible for the RT instabilities and associated vorticity observed even at the earliest stages of the flow. Instabilities of the Kelvin-Helmholtz (KH) type and 'roll-up' phenomena also arise from the viscosity contrasts present and these promote mixing of the two fluids, which is counteracted by the gravitational flow proportional to $g \cos \theta$ that promote segregation. It is this mixing, enhanced by the presence of instabilities, that is responsible for the slope of the $M_{0.95} / M_{0}$ curves being greater than $-H / L$ during the linear stage for $t \leq 25$ in Fig. 2a.

At the latter stages of the flow (i.e. for $t \geq 25$ for this set of parameters), it can be seen that the large majority of fluid ' 1 ' has been driven out of the channel, as shown in Fig. 3. The remnants of fluid ' 1 ' assume the form of thin layers adjacent to the upper and lower walls. The layer at the lower wall is thicker than that at the upper one since the denser, fluid ' 1 ' is expected to settle on the lower channel wall. The flow at these relatively late stage is expected to be dominated by diffusion. It is this change in the character of the flow, from intensely convective to diffusive, that is primarily responsible for the change in the slope of the $M_{0.95} / M_{0}(t)$ plot for $t \geq 25$ in Fig. $2 \mathrm{~b}$.

Fig. 4 shows the evolution of vorticity (top) and velocity (bottom) for the same parameter values as those used to generate Fig. 3. It can be seen that for $15 \leq t \geq 25$ the central region contains intense mixing and strong vortical activity. At later time, for $t \geq 30$, it can be seen that the level of vorticity is very low in the central region and this coincides with the occurrence of a two-layer structure in this region; this as can be seen in Fig. 5, which presents an enlarged version of Fig. 4 for $t=35$ and $15 \leq x \leq 25$. Vortical structures can be seen in the inlet region at all times because of the flow due to the applied pressure-gradient there.

We have also plotted the streamwise variation of the depth-averaged concentration, $\bar{c}(x, t) \equiv \int_{0}^{1} c d y$ for the same parameters as those used to generate Fig. 3. Note that we have rescaled the streamwise co-ordinate as $(x-t) / t^{1 / 2}$ in an effort to 'subtract' the 
background pressure driven flow. The collapse of the plots on a single curve demonstrates that the flow remaining following this 'subtraction' is diffusive in nature [46].

Next, we study the effect of inclination angle, $\theta$, on the displacement characteristics with $R e=S c=200, m=2$ and $r=2$. As shown in Fig. 7a, increasing the degree of channel inclination by increasing the value of $\theta$ progressively from $\theta=0$ to $\theta=90^{\circ}$ leads to more rapid displacement in comparison to the horizontal channel case; although, interestingly, the displacement curve for $\theta=30^{\circ}$ and $\theta=60^{\circ}$ are almost identical. In contrast, declining the channel retards the displacement process, which appears to be weakly dependent on the value of $\theta$ for $\theta<0$. It can be seen that all the curves in Fig. 7a lie below $1-t H / L$ which corresponds to plug flow displacement, which is due to the presence of instabilities which enhance mixing; the curves closest to the $1-t H / L$ line are those associated with $\theta<0$. In Fig. 7b, it is clearly seen that increasing $\theta$ leads to an increase in the speed of the front.

The results depicted in Fig. 7 are rationalised by examining the effect of varying $\theta$ on the concentration contours at $t=20$ and with all other parameters remaining fixed; this is shown in Fig. 8. In the $\theta=0^{\circ}$ case, fluid ' 1 ' is penetrated by a 'finger' of fluid ' 2 ' with a blunt 'nose' separating the two fluids. This finger is asymmetric: the layer of fluid ' 1 ' adjoining the lower wall, left behind by the penetrating finger, is far thicker than its counterpart at the upper wall. The observed asymmetry is brought about by the density contrast, characterised by $r=2$. The 'interfaces' separating the two fluids are not sharp, having being smeared by diffusion.

In contrast with the $\theta=0^{\circ}$ case, for positive inclination angles, the flow appears to be considerably more unstable. The RT and KH instabilities accompanying the flow in this case engender a significant level of mixing. As a result, the region separating fluids ' 1 ' and ' 2 ' is highly diffuse and it is difficult to identify a finger-like structure, such as the one exhibited by the $\theta=0^{\circ}$ case. The fraction of the channel occupied by regions of pure, unmixed fluid ' 1 ' decreases with increasing $\theta$, due to the diminishing role of the gravitational forces proportional to $g \cos \theta$ that act to drive fluid segregation; this indicates that the removal rate of fluid ' 1 ' from the channel increases with $\theta$. A comparison of these dynamics with that for $\theta<0$ shows that, due to the absence of a RT destabilising mechanism, the flow in the latter case is not accompanied by instabilities and the two fluids are separated by relatively sharp front. Furthermore, the flow characteristics and displacement rates appear to be insensitive to variations in $\theta$. These observations are in line with the results presented 


\section{in Fig. 7.}

In Fig. 9, we show the effect of varying $\theta$ on the streamwise variation of $\bar{c}$ in rescaled co-ordinates. It is clearly seen that the flow remaining following the 'subtraction' of the pressure-driven component is diffusive, as demonstrated by the collapse of the plots on a single curve. The collapse is particularly good for $\theta<0$, which, as shown in Fig. 8, are associated with weak instabilities.

In order to estimate the relative influence of the viscosity contrast on the dynamics, we show the results of setting $m=1$ in Figs 10 and 11 and the rest of the parameter values are the same as in Fig. 7. A comparison of Figs. 8 and 11 reveals that for this set of parameter values, which reflect the absence of viscosity contrasts, RT instabilities grow along the 'interface' between the two fluids, enabling a strong transverse mixing and rapid displacement rates that are very similar to the $m=2$ case; these effects decrease with decreasing $\theta$ and for $\theta<0$, which is also similar to $m=2$ case. The results suggest that the flow characteristics are dominated by density contrasts.

The effect of varying $r$ on the dynamics is investigated next. As shown in Fig. 12(a) and (b), increasing the value of $r$ leads to an increase in both the displacement rate and front velocity. This is as expected since increasing the density contrast promotes the development of RT instabilities, which, in turn, engender rapid fluid mixing. In Fig. 12(c) and (d), we demonstrate that raising the value of $F r$, which corresponds to increasing the relative significance of gravitational forces, accelerates the removal of fluid ' 1 ' and diminishes the duration during which the flow is dominated by diffusion.

We have also examined the case of fluid ' 1 ' displaced by a less viscous yet denser fluid ' 1 '; this is characterised by $r=0.5$ with the rest of the parameters remaining unchanged from Figs. 7 and 8. As can be ascertained upon inspection of Figs. 13 and 14, the trends are essentially the opposite of those associated with the $r=2$ case. In the horizontal channel case, a reasonably well-defined, asymmetric fluid ' 2 ' finger can be seen in Fig. 14, with the upper wall layer of fluid ' 1 ' being thicker than that adjoining the lower wall. Due to the absence of a RT mechanism for $\theta>0$ in this case since $r<1$, the displacement of fluid ' 1 ' by the denser fluid ' 2 ' is relatively stable. Mixing in inclined channels for this case occurs by a $\mathrm{KH}$ mechanism, as evidenced by the 'roll-up' events seen for $\theta=30^{\circ}$ and $60^{\circ}$ in Fig. 14 in particular, and by diffusion. The tiny tip of the 'finger' for $\theta=90^{\circ}$ was observed in the experiment of miscible displacement in Hele-Shaw cell [39]. For $\theta<0$, RT instabilities lead 
to vigorous fluid mixing and rapid displacement rates, which are maximised at $\theta=-90^{\circ}$. The trends observed in the streamwise variation of the depth-averaged concentration for varying $\theta$ are also essentially the opposite of those seen in Fig. 9; this is shown in Fig. 15. Inspection of Figs. 2(b), $7(\mathrm{~b}), 10(\mathrm{~b})$ and $13(\mathrm{~b})$ reveals that $V_{\text {tip }}=\mathrm{d} x_{\text {tip }} / \mathrm{d} t$ increases with increase in angle, $\theta$. However, it appears that $V_{\text {tip }}$ is essentially constant due to the pressure-driven flow in all the cases examined.

\section{CONCLUSIONS}

We have investigated the pressure-driven displacement of two miscible fluids in inclined channels in the presence of viscosity and density gradients. Direct numerical simulations of the flow have been carried out via solution of the continuity and Navier-Stokes equations, without the Boussinesq approximation, coupled to a convective-diffusion equation for the concentration of the more viscous fluid through a concentration-dependent viscosity and density. We have examined the effect of density ratio, Froude number, and channel inclination on the flow dynamics for moderate Reynolds numbers and viscosity ratios, focusing on the case wherein the displaced fluid is also the more viscous one. Our results indicate that the rates of mixing and displacement of the more viscous fluid, promoted by the development of Rayleigh-Taylor instabilities, are enhanced with increasing density ratio and Froude number. Furthermore, these rates are shown to increase (decrease) with increasing (decreasing) inclination angles when the displaced fluid is also the denser (lighter) one.

\section{Acknowledgments}

We acknowledge support from the Engineering and Physical Sciences Research Council (through grant number EP/D503051/1), and the Department of Trade and Industry of the UK.

[1] D. D. Joseph, R. Bai, K. P. Chen, and Y. Y. Renardy, "Core-annular flows," Ann. Rev. Fluid Mech. 29, 65 (1997). 
[2] Q. Cao, L. Ventresca, K. R. Sreenivas, and A. K. Prasad, "Instability due to viscosity stratification downstream of a centreline injector," Can. J. Chem. Eng. 81, 913 (2003).

[3] M. Regner, M. Henningsson, J. Wiklund, K. Östergren and C. Trägårdh, "Predicting the displacement of yoghurt by Water in a pipe using CFD," Chem. eng. Technology 30, 844 (2007).

[4] D. D. Joseph and Y. Y. Renardy, Fundamentals of Two-Fluid Dynamics. Part I: Mathemtical Theory and Applications (Springer-Verlag, New York, 1992).

[5] C. S. Yih, "Instability due to viscous stratification," J. Fluid Mech. 27, 337 (1967).

[6] A. P. Hooper and W. G. C. Boyd, "Shear flow instability at the interface between two fluids," J. Fluid Mech. 128, 507 (1983).

[7] E. J. Hinch, "A note on the mechanism of the instability at the interface between two shearing fluids," J. Fluid Mech. 144, 463 (1984).

[8] S. G. Yiantsios and B. G. Higgins, "Numerial solution of eigenvalue problems using the compound matrix-method," J. Comp. Phys. 74, 25 (1988).

[9] M. K. Smith, "The mechanism for the long-wave instability in a thin liquid film," J. Fluid Mech. 217, 469 (2001).

[10] P. A. M. Boomkamp and R. H. M. Miesen, "Classification of instabilities in parallel two-phase flow," Int. J. Multiphase Flow 22, 67 (1996).

[11] F. Charru and E. J. Hinch, "Phase diagram' of interfacial instabilities in a two-layer Couette flow and mechanism for the long-wave instability," J. Fluid Mech. 414, 195 (2000).

[12] K. C. Sahu, P. Valluri, P. D. M. Spelt, and O. K. Matar, "Linear instability of pressure-driven channel flow of a Newtonian and Herschel-Bulkley fluid," Phys. Fluids 19, 122101 (2007).

[13] D. D. Joseph, M. Renardy, and Y. Y. Renardy, "Instability of the flow of two immiscible liquids with different viscosities in a pipe," J. Fluid Mech. 141, 309 (1984).

[14] Y. Y. Renardy and D. D. Joseph, "Couette-flow of two fluids between concentric cylinders," J. Fluid Mech. 150, 381 (1985).

[15] H. H. Hu and D. D. Joseph, "Lubricated pipelining: stability of core-annular flows. Part 2," J. Fluid Mech. 205, 395 (1989).

[16] L. Preziosi, K. Chen, and D. D. Joseph, "Lubricated pipelining: stability of core-annular flow," J. Fluid Mech. 201, 323 (1989).

[17] H. H. Hu, T. S. Lundgren, and D. D. Joseph, "Stability of core-annular flow with a small 
viscosity ratio," Phys. Fluids A 2, 1945 (1990).

[18] C. E. Hickox, "Instability due to viscosity and density stratification in axisymmetric pipe flow," Phys. Fluids 14, 251 (1971).

[19] H. H. Hu and N. Patankar, "Non-axisymmetric instability of core-annular flow," J. Fluid Mech. 290, 213 (1995).

[20] M. E. Charles, G. W. Govier, and G. W. Hodgson, "The horizontal pipeline flow of equal density oil-water mixtures," Can. J. Chem. Eng. 39, 27 (1961).

[21] R. Bai, K. Chen, and D. D. Jospeh, "Lubricated pipelining: stability of core annular flow. Part 5. Experiments and comparison with theory," J. Fluid Mech. 240, 97 (1992).

[22] J. Li and Y. Y. Renardy, "Direct simulation of unsteady axisymmetric core-annular flow with high viscosity ratio," J. Fluid Mech. 391, 123 (1999).

[23] C. Kouris and J. Tsamopoulos, "Dynamics of axisymmetric core-annular flow in a straight tube. I. The more viscous fluid in the core, bamboo waves," Phys. Fluids 13, 841 (2001).

[24] C. Kouris and J. Tsamopoulos, "Dynamics of axisymmetric core-annular flow in a straight tube. II. The less viscous fluid in the core, saw tooth waves," Phys. Fluids 14, 1011 (2002).

[25] C. Kouris and J. Tsamopoulos, "Core-annular flow in a periodically constricted circular tube. Part 1. Steady-state linear stability and energy analysis," J. Fluid Mech. 432, 31 (2001).

[26] C. Kouris and J. Tsamopoulos, "Core-annular flow in a periodically constricted circular tube. Part 2. Nonlinear dynamics," J. Fluid Mech. 470, 181 (2002).

[27] H. H. Wei and D. S. Rumschitzki, "The linear stability of a core-annular flow in an asymptotically corrugated tube," J. Fluid Mech. 466, 113 (2002).

[28] H. H. Wei and D. S. Rumschitzki, "The weakly nonlinear interfacial stability of a core-annular flow in a corrugated tube," J. Fluid Mech. 466, 149 (2002).

[29] B. T. Ranganathan and R. Govindarajan, "Stabilisation and destabilisation of channel flow by location of viscosity-stratified fluid layer," Phys. Fluids. 13(1), 1 (2001).

[30] R. Govindarajan, "Effect of miscibility on the linear instability of two-fluid channel flow," International J. Multiphase Flow 30, 1177 (2004).

[31] P. Ern, F. Charru, and P. Luchini, "Stability analysis of a shear flow with strongly stratified viscosity," J. Fluid Mech. 496, 295 (2003).

[32] G. I. Taylor, "Deposition of viscous fluid on the wall of a tube," J. Fluid Mech. 10, 161 (1961).

[33] B. G. Cox, "On driving a viscous fluid out of a tube," J. Fluid Mech. 14, 81 (1962). 
[34] P. Petitjeans and T. Maxworthy, "Miscible displacements in capillary tubes. Part 1. Experiments," J. Fluid Mech. 326, 37 (1996).

[35] C.-Y. Chen and E. Meiburg, "Miscible displacement in capillary tubes. Part 2. Numerical simulations," J. Fluid Mech. 326, 57 (1996).

[36] J. Kuang, T. Maxworthy, and P. Petitjeans, "Miscible displacements between silicone oils in capillary tubes," Eur. J. Mech. 22, 271 (2003).

[37] R. Balasubramaniam, N. Rashidnia, T. Maxworthy, and J. Kuang, "Instability of miscible interfaces in a cylindrical tube," Phys. Fluids 17, 052103 (2005).

[38] E. Lajeunesse, J. Martin, N. Rakotomalala, and D. Salin, "3D instability of miscible displacements in a Hele-Shaw cell," Phys. Rev. Lett. 79, 5254 (1997).

[39] E. Lajeunesse et al., "Miscible displacement in a Hele-Shaw cell at high rates," J. Fluid Mech. 398, 299 (1999).

[40] J. Scoffoni, E. Lajeunesse, and G. M. Homsy, "Interface instabilities during displacement of two miscible fluids in a vertical pipe," Phys. Fluids 13, 553 (2001).

[41] C. Gabard and J.-P. Hulin, "Miscible displacement of non-Newtonian fluids in a verticl tube," Eur. Phys. J. E. 11, 231 (2003).

[42] M. d'Olce et al., "Pearl and mushroom instability patterns in two miscible fluids' core annular flows," Phys. Fluids 20, 024104 (2008).

[43] B. Sevlam, S. Merk, R. Govindarajan, and E. Meiburg, "Stability of miscible core-annular flows with viscosity stratification," J. Fluid Mech. 592, 23 (2007).

[44] S. V. Malik and A. P. Hooper, "Linear stability and energy growth of viscosity stratified flows," Phys. Fluids 17, 024101 (2005).

[45] M. H. I. Baird et al., "Unsteady axial mixing by natural convection in a vertical column," AIChE J. 38, 1825 (1992).

[46] M. Debacq et al., "Self-similar concentration profiles in buoyant mixing of miscible fluids in a vertical tube," Phys. Fluids 13, 3097 (2001).

[47] T. Séon et al., "Buoyant mixing of miscible fluids in tilted tubes," Phys. Fluids 16, L103 (2004).

[48] T. Séon et al., "LIF measurements of buoyancy driven mixing in tilted tubes," Phys. Fluids 18, 041701 (2006).

[49] T. Séon et al., "Buoyancy driven front dynamics in tilted tubes," Phys. Fluids 17, 031702 
(2005).

[50] T. Séon et al., "Front dynamics and macroscopic diffusion in buoyant mixing in a tilted tube.," Phys. Fluids 19 (12), 125105 (2007).

[51] J. Fernandez, P. Kurowski, P. Petitjeans, and E. Meiburg, "Density driven unstable flows of miscible fluids in a Hele-Shaw cell,” J. Fluid Mech. 451, 239 (2002).

[52] E. J. Strang and H. J. S. Fernando, "Entrainment and mixing in stratified shear flows," J. Fluid Mech. 428, 349 (2001).

[53] O. Pouliquen, J. M. Chomaz, and P. Huerre, "Propagating Homboe waves at the interface between two immiscible fluids," J. Fluid Mech. 266, 277 (1994).

[54] T. Séon et al., "Transient buoyancy-driven front dynamics in nearly horizontal tubes," Phys. Fluids 19, 123603 (2007).

[55] Y. Hallez and J. Magnaudet, "Effects of channel geometry on buoyancy-driven mixing," Phys. Fluids 20, 053306 (2008).

[56] K. C. Sahu, H. Ding, P. Valluri, and O. K. Matar, "Linear stability analysis and numerical simulation of miscible channel flows," , submitted to Phys. Fluids.

[57] H. Ding, P. D. M. Spelt, and C. Shu, "Diffuse interface model for incompressible two-phase flows with large density ratios," J. Computational Physics 226, 2078 (2007).

[58] H. Ding and P. D. M. Spelt, "Wetting condition in diffuse interface simulations of contact line motion," Phys. Rev. E 75, 046708 (2007).

[59] H. Ding and P. D. M. Spelt, "Inertial effects in droplet spreading: a comparison between diffuse-interface and level-set simulations," J. Fluid Mech. 576, 287 (2007).

[60] H. Ding and P. D. M. Spelt, "Onset of motion of three-dimensional droplet on a wall in a shear flow at moderate Reynolds numbers," J. Fluid Mech. 599, 341 (2008). 


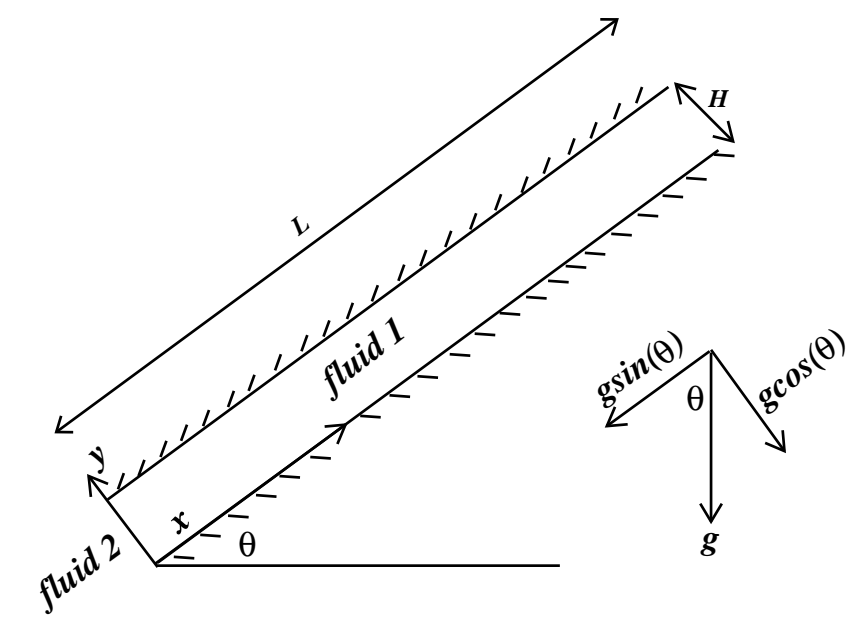

FIG. 1: Schematic diagram showing the initial flow configuration: fluid '1' occupies the entire channel, inclined at an angle $\theta$ to the horizontal, and is about to be displaced by fluid ' 2 '. 
(a)

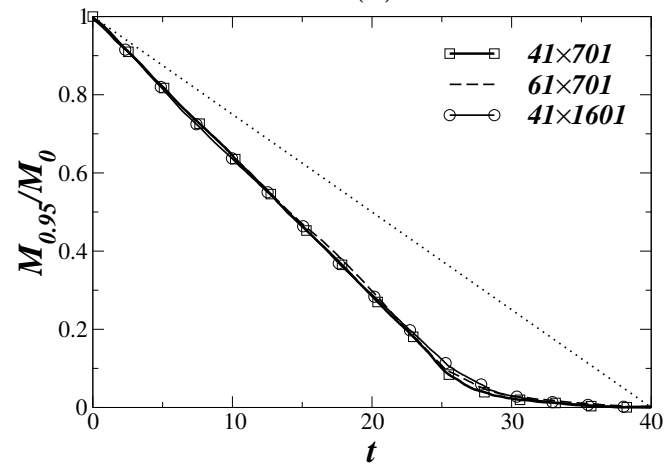

(b)

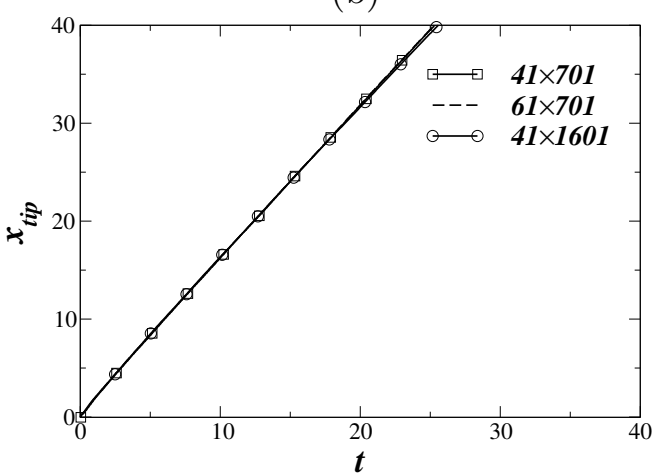

FIG. 2: Mass fraction of the displaced fluid $M_{0.95} / M_{0}$, (a), and temporal evolution of the position of the leading 'front' separating the two fluids, $x_{\text {tip }}$, (b), obtained using different mesh densities for $R e=200, S c=100, F r=0.316, m=2, r=1.5$, and $\theta=30^{\circ}$. The dotted line in panel (a) represents the limiting case given by $M_{0.95} / M_{0}=1-t H / L$. This corresponds to the case wherein fluid ' 1 ' is displaced by fluid ' 2 ' in plug flow and prior to the sharp, vertical interface separating the two fluids exiting the channel. 

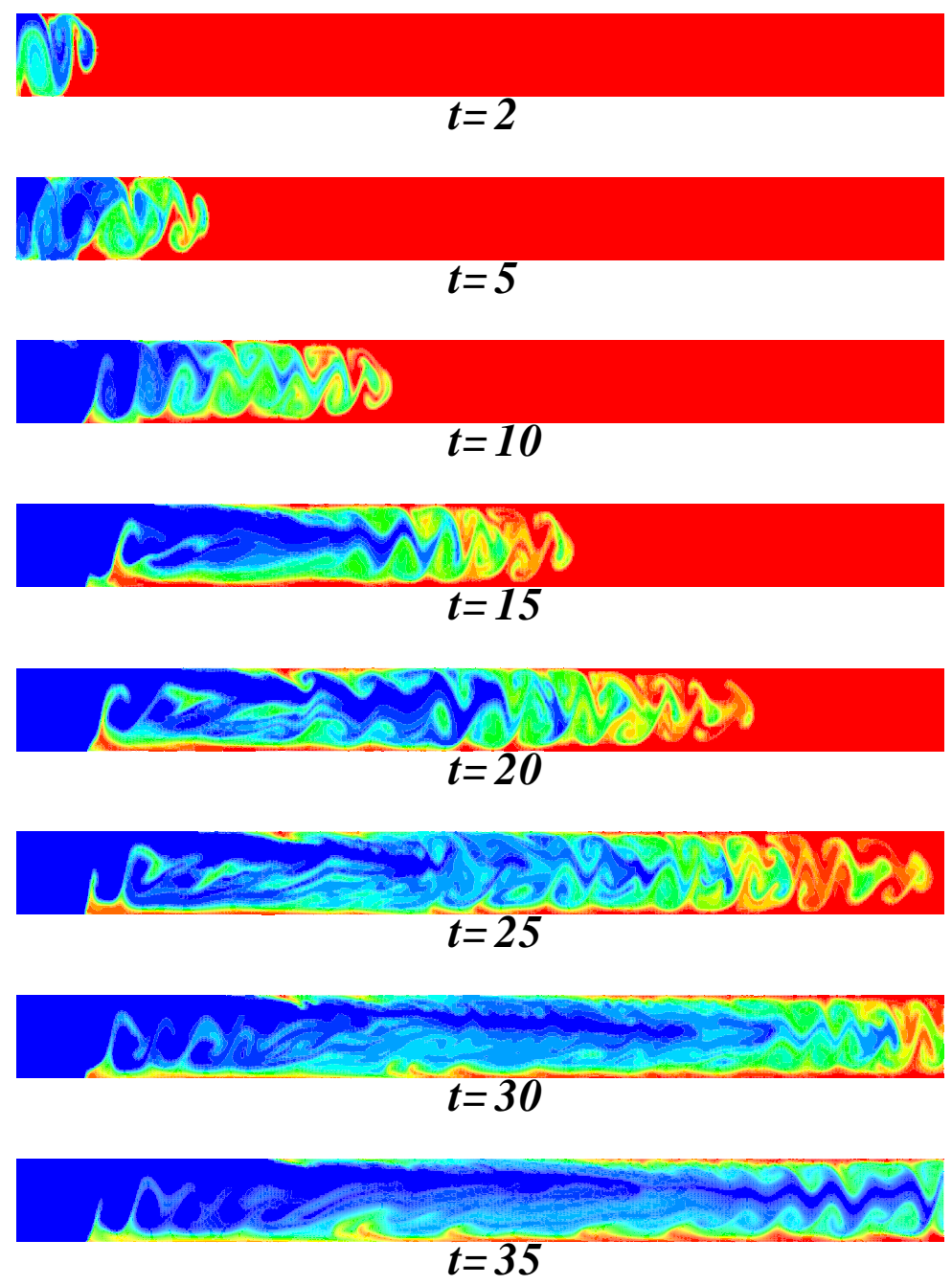

FIG. 3: Spatio-temporal evolution of the concentration contours for the same parameters as those used to generate Fig. 2. 

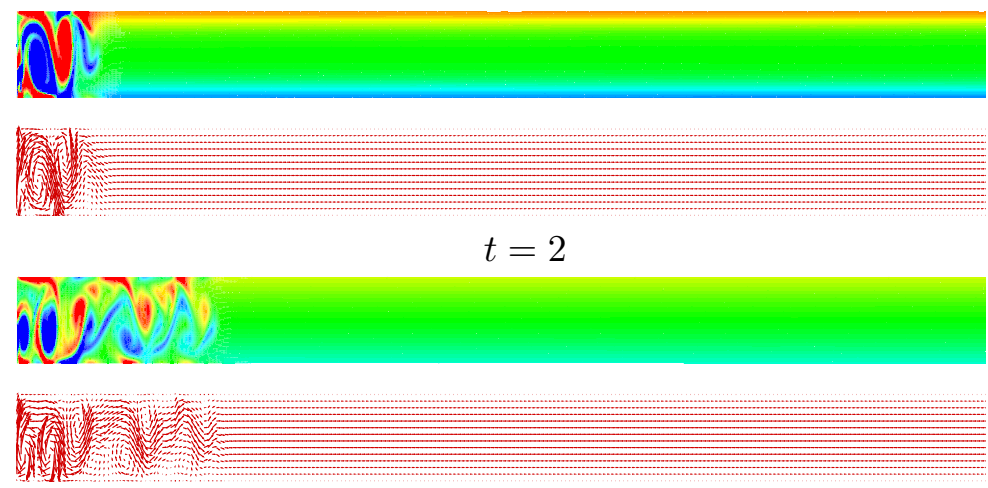

$t=5$
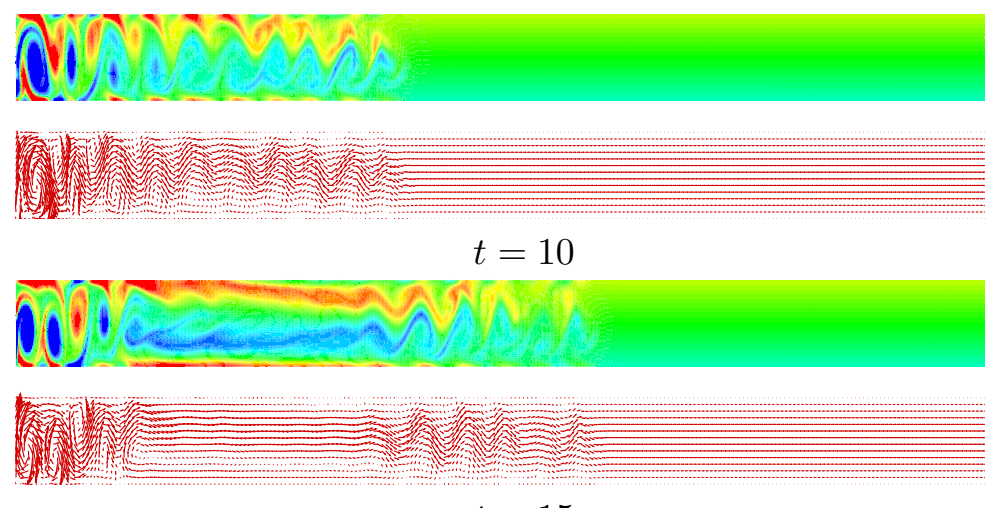

$$
t=15
$$
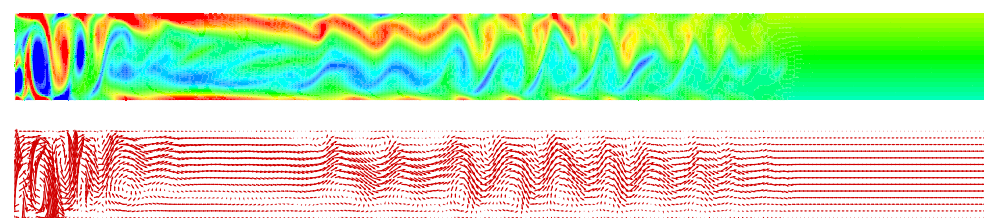

$$
t=20
$$
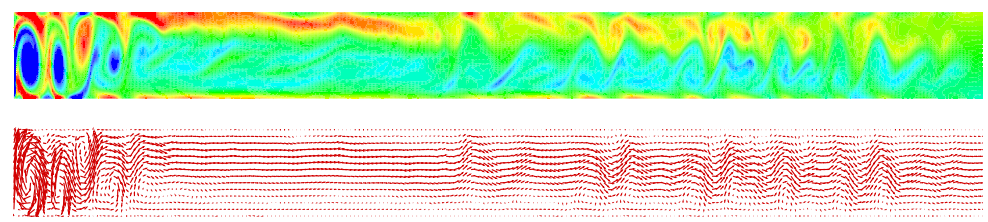

$$
t=25
$$
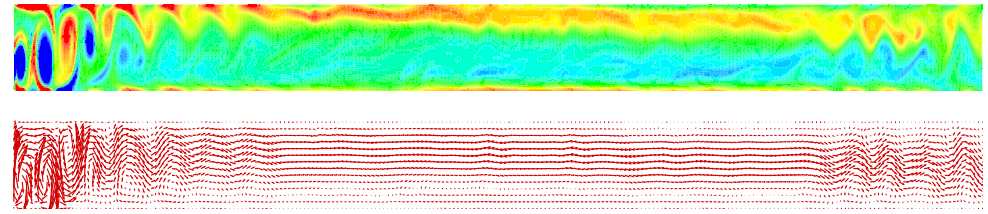

$$
t=30
$$

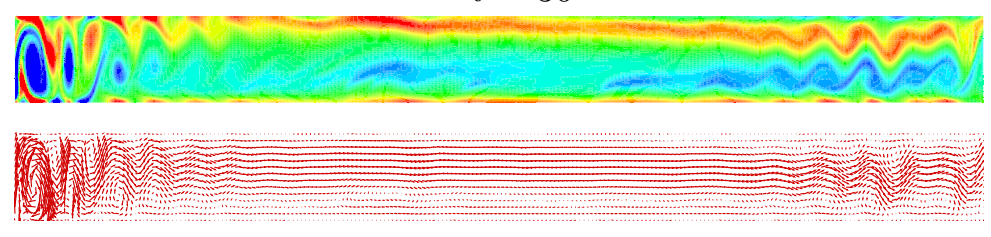

$$
t=35
$$

FIG. 4: Evolution of the vorticity and velocity vectors for the same parameter values as those used to generate Fig. 3. 


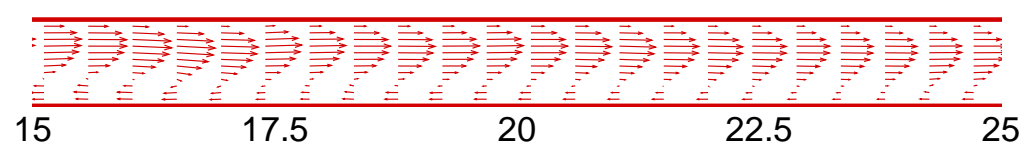

FIG. 5: An enlarged view of velocity vectors of Fig 4 at $\mathrm{t}=35$ and $15 \leq x \leq 25$. 


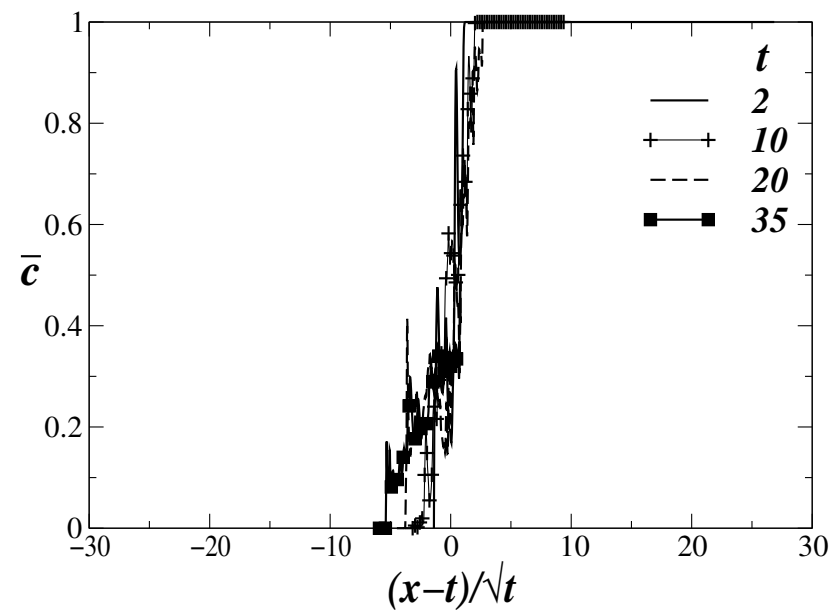

FIG. 6: Streamwise variation of the depth-averaged concentration, $\bar{c} \equiv \int_{0}^{1} c d y$, for the same parameters as those used to generate Fig. 3. Here, the $x$-coordinate has been rescaled to $(x-t) / t^{1 / 2}$. 
(a)

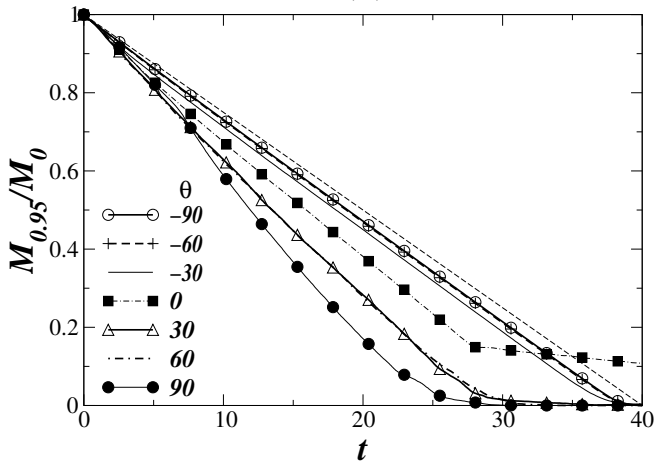

(b)

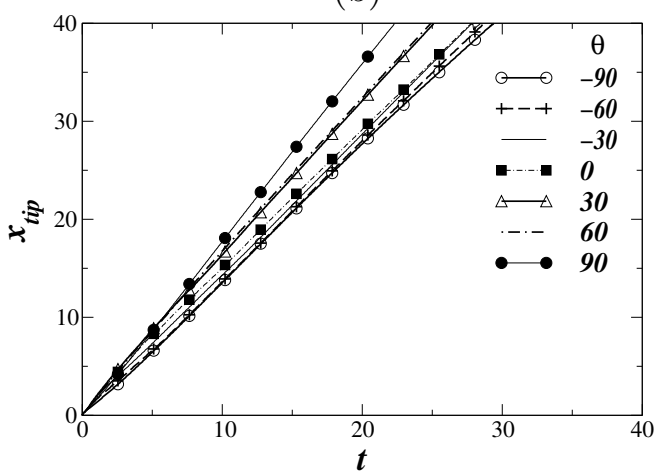

FIG. 7: Effect of channel inclination, $\theta$, on the mass fraction of the displaced fluid '1', (a), the temporal evolution of the position of the leading 'front' separating the two fluids $x_{\text {tip }}$, (b). The rest of the parameter values are $R e=200, F r=0.316, S c=100, m=2$, and $r=2$. The dotted line in panel (a) is the analogue of that shown in Fig. 2a. 


$$
\theta=0^{\circ}
$$

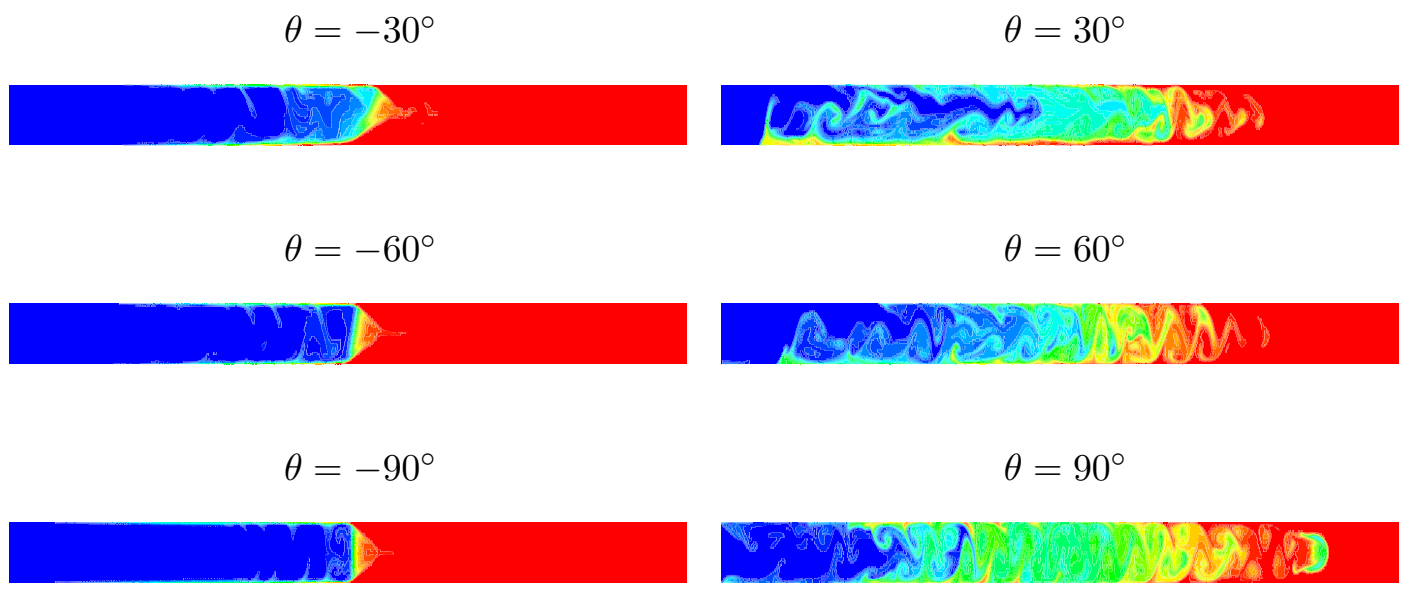

FIG. 8: The effect of inclination angle, $\theta$, on the concentration contours at $t=20$. The rest of the parameter values remain unchanged from those used to generate Fig. 7. 

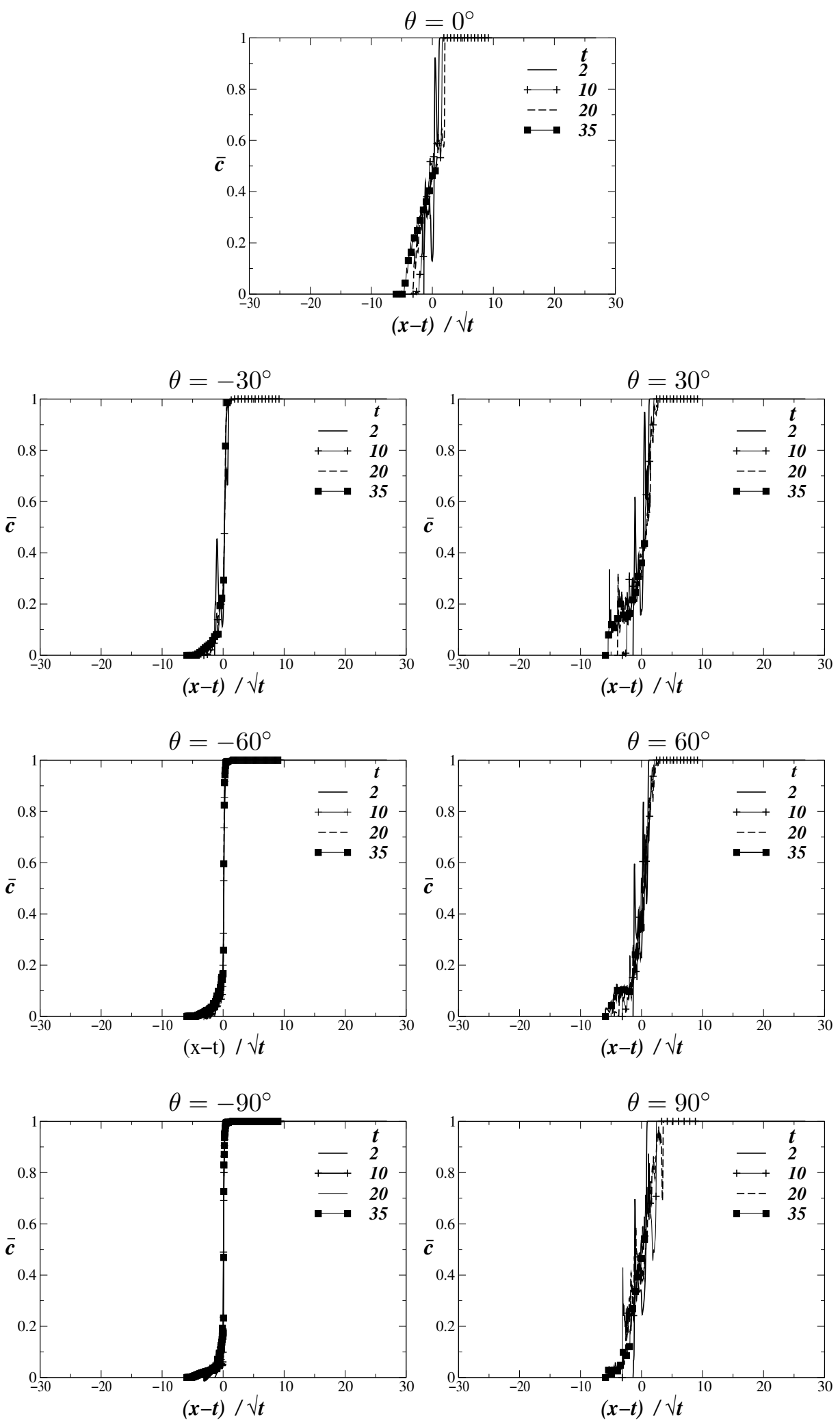

FIG. 9: Streamwise variation of the depth-averaged concentration, $\bar{c} \equiv \int_{0}^{1} c d y$, for the same parameters as those used to generate Fig. 7 . Here, the $x$-coordinate has been rescaled to $(x-t) / t^{1 / 2}$. 
(a)

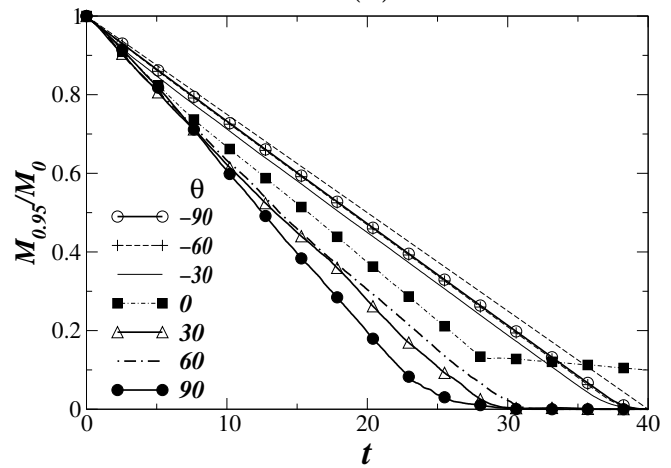

(b)

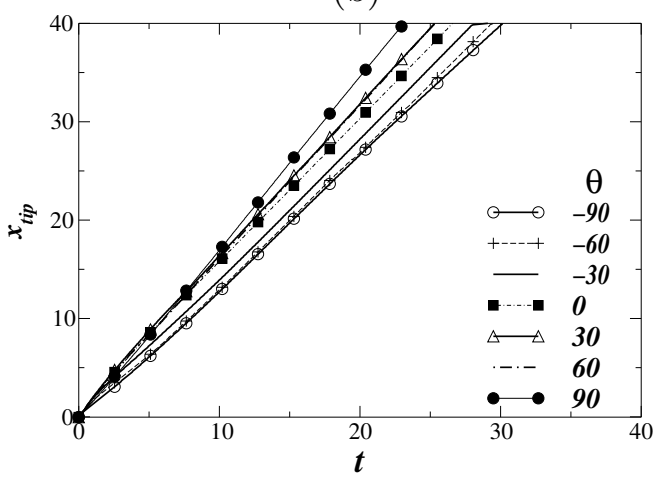

FIG. 10: Effect of channel inclination, $\theta$, on the mass fraction of the displaced fluid ' 1 ', (a), the temporal evolution of the position of the leading 'front' separating the two fluids $x_{\mathrm{tip}}$, (b), for $m=1$. The rest of the parameter values are $R e=200, F r=0.316, S c=100$, and $r=2$. The dotted line in panel (a) is the analogue of those shown in Figs. 2a and 7a. 


$$
\theta=0^{\circ}
$$

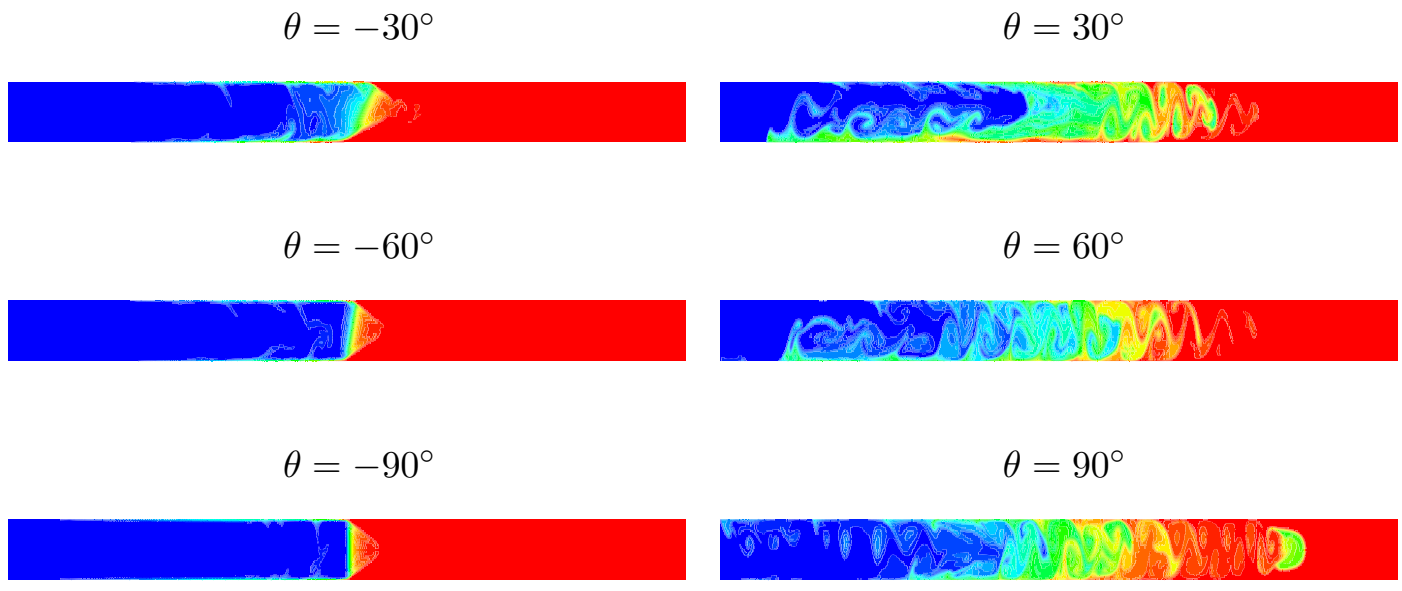

FIG. 11: The effect of inclination angle, $\theta$, on the concentration contours at $t=20$ for $m=1$. The rest of the parameter values remain unchanged from those used to generate Fig. 10. 
(a)

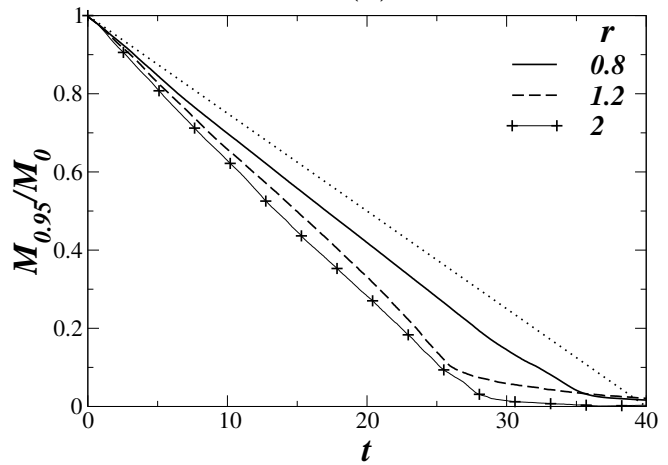

(c)

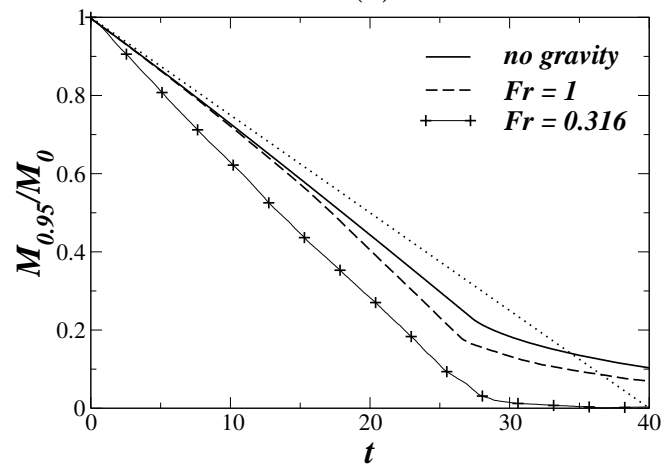

(b)

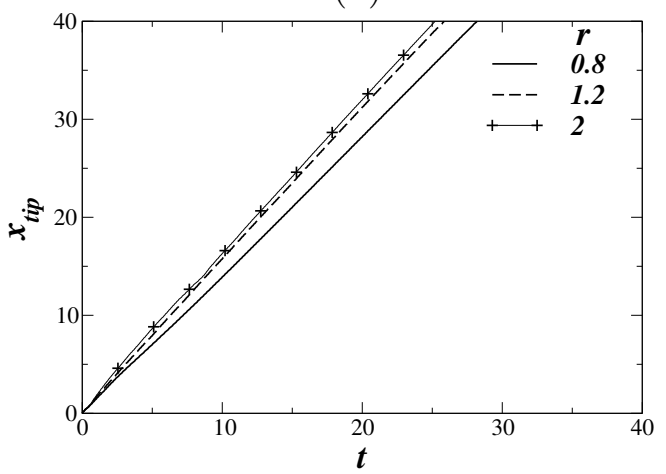

(d)

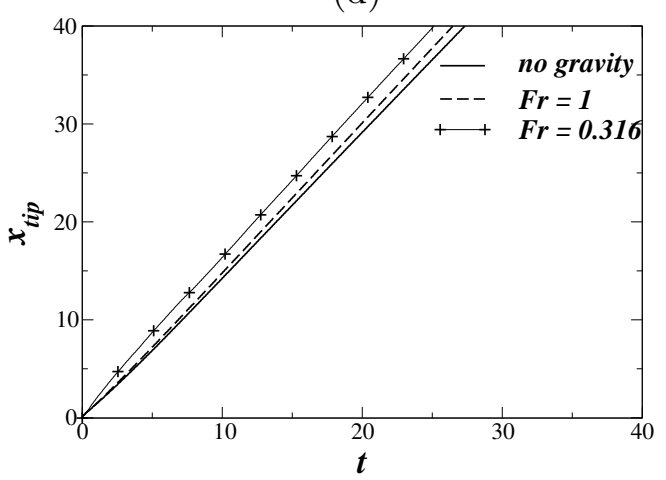

FIG. 12: Effect of density ratio, $r$, and Froude number, $F r$, on the mass fraction of the displaced fluid ' 1 ', (a) and (c), the temporal evolution of the position of the leading 'front' separating the two fluids $x_{\text {tip }}$, (b) and (d). The rest of the parameter values remain unchanged from those used to generate Fig. 2. The dotted lines in (a) and (c) are analogues of those shown in Figs. 2a, 7a and 10a. The lines associated with the 'no gravity' legend correspond to the case wherein the gravitational terms in the governing equations have been neglected. 
(a)

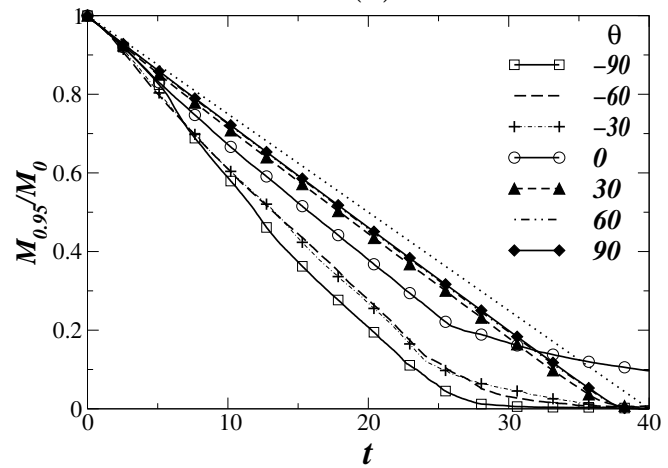

(b)

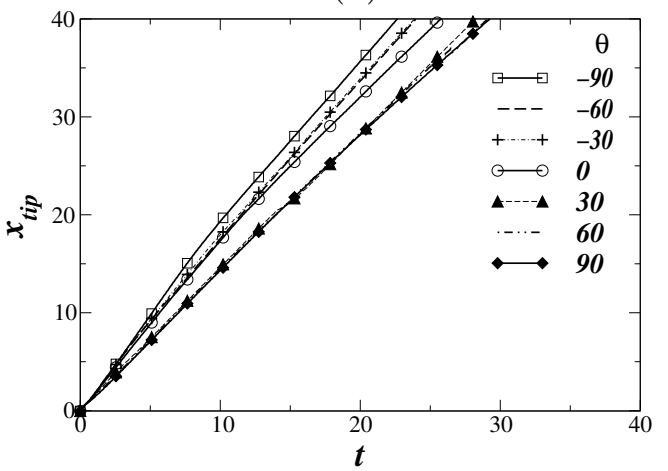

FIG. 13: Effect of channel inclination, $\theta$, on the mass fraction of the displaced fluid ' 1 ', (a), the temporal evolution of the position of the leading 'front' separating the two fluids $x_{\text {tip }}$, (b). Here, $r=0.5$ and the rest of the parameter values remain unchanged from those used to generate Fig. 7. The dotted line in (a) is analogues of those shown in Figs. 2a, 7a, 10a, and 12(a) and (c). 


$$
\theta=0^{\circ}
$$

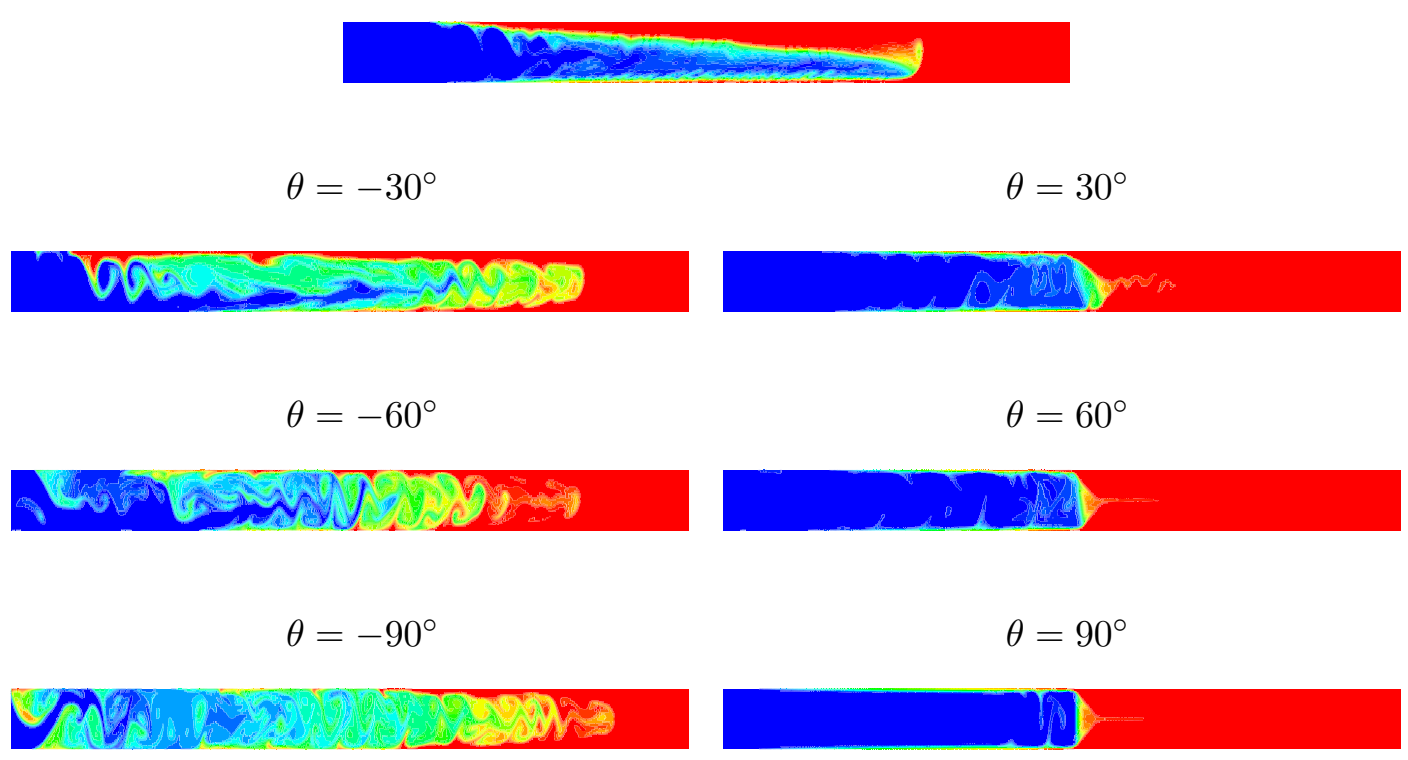

FIG. 14: The effect of inclination angle, $\theta$, on the concentration contours at $t=20$. The rest of the parameter values remain unchanged from those used to generate Fig. 13. 

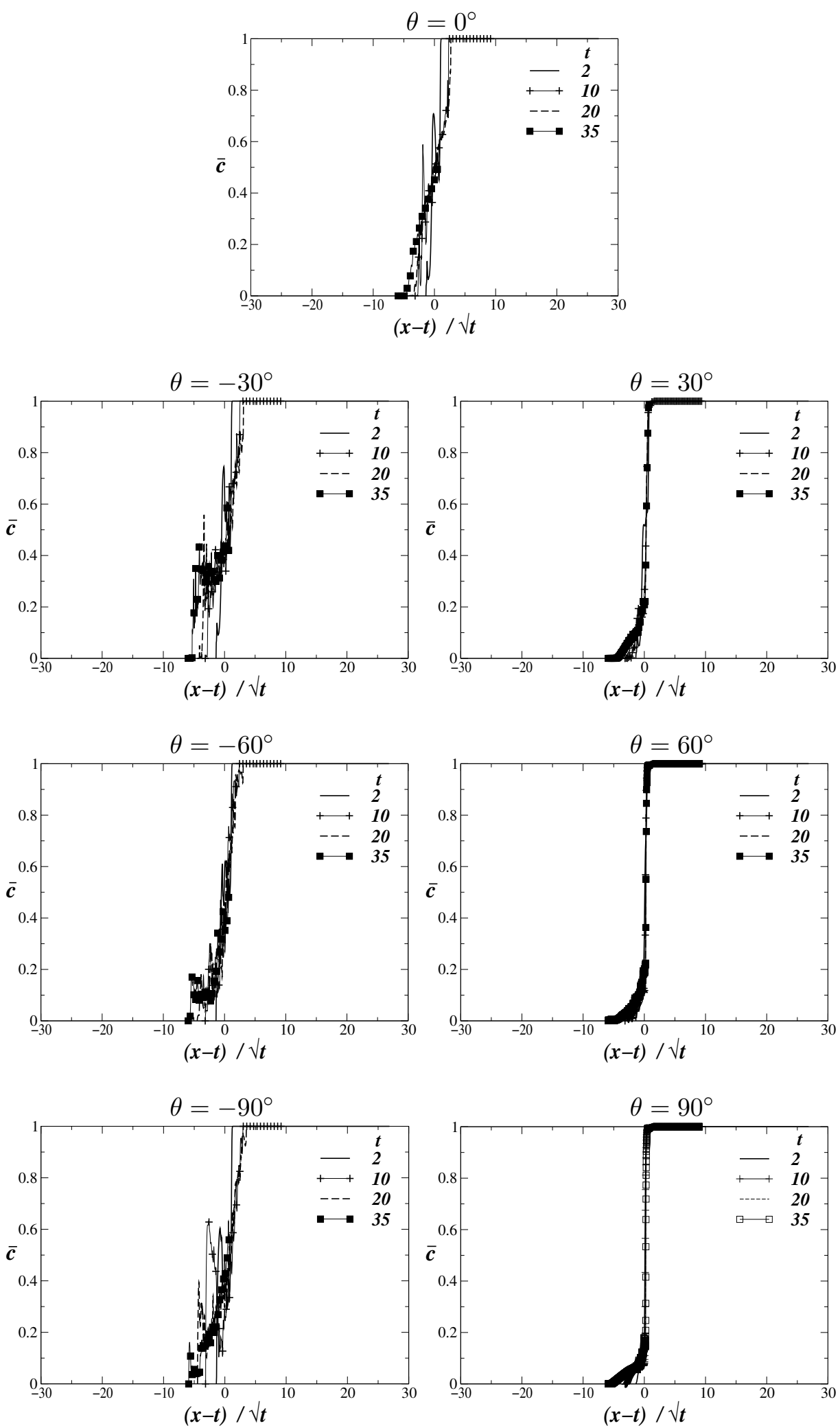

FIG. 15: Streamwise variation of the depth-averaged concentration, $\bar{c} \equiv \int_{0}^{1} c d y$, for the same parameters as those used to generate Fig. 13. Here, the $x$-coordinate has been rescaled to ( $x-$ t)/ $t^{1 / 2}$. 